
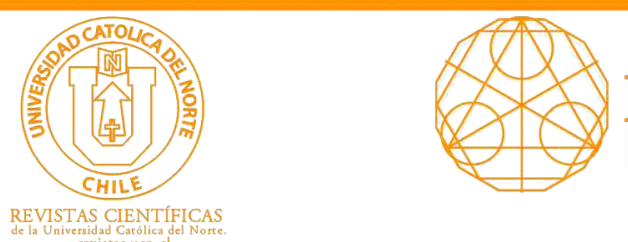

dof) 10.22199 /issn.0717-6279-2020-04-0066

\title{
Quasi self-dual codes over non-unital rings of order six*
}

Adel Alahmadi ${ }^{1}$ (1) orcid.org/0000-0002-7758-3537

Amani Alkathiry² (0) orcid.org/0000-0001-7920-859X

Alaa Altassan ${ }^{3}$ (0) orcid.org/0000-0003-2729-7396

Widyan Basaffar ${ }^{4}$ [D orcid.org/0000-0003-1639-9186

Alexis Bonnecaze ${ }^{5}$ (1) orcid.org/0000-0002-9642-7222

Hatoon Shoaib ${ }^{6}$ (1) orcid.org/0000-0002-1888-8154

Patrick Solé ${ }^{7}$ (D) orcid.org/0000-0002-4078-8301

King Abdulaziz University, Dept. of Mathematics, Jeddah, Saudi Arabia.

1曰adelnife2@yahoo.com ; ${ }^{-1}$ aaltassan@kau.edu.sa; ${ }^{-}$whbasaffar@kau.edu.sa ;

6-hashoaib@kau.edu.sa

${ }^{2}$ Umm Al-Qura University, Dept. of Mathematics, Makkah, Saudi Arabia

-aakathiry@uqu.edu.sa

Aix-Marseille Université ,CNRS, Marseille, France.

5曰alexis.bonnecaze@univ-amu.fr; 7 sole@enst.fr

Received: February 2020 | Accepted: June 2020

\section{Abstract:}

There exist two semi-local rings of order 6 without identity for the multiplication. We classify up to coordinate permutation self-orthogonal codes of length $n$ and size $6^{n / 2}$ over these rings (called here quasi self-dual codes or $Q S D$ ) till the length $n=8$. To any such code is attached canonically a $\mathbb{Z}_{6}$-code, which, when self-dual, produces an unimodular lattice by Construction A.

Keywords: Non-unital rings; Semi-local rings; Self-orthogonal codes; Unimodular lattices.

MSC (2020): 94B05, 16A10.

\section{Cite this article as (IEEE citation style):}

A. Alahmadi, A. Alkathiry, A. Altassan, W. Basaffar, A. Bonnecaze, H. Shoaib, and P. Solé, "Quasi self-dual codes over non-unital rings of order six", Proyecciones (Antofagasta, On line), vol. 39, no. 4, pp. 1083-1095, Aug. 2020, doi: 10.22199/issn.07176279-2020-04-0066.

Article copyright: (c) 2020 Adel Alahmadi, Amani Alkathiry, Alaa Altassan, Widyan Basaffar, Alexis Bonnecaze, Hatoon Shoaib and Patrick Solé. This is an open access article distributed under the terms of the Creative Commons License, which permits unrestricted use and distribution provided the original author and source are credited.

*A preprint of this paper can be found at Archive ouverte HAL 


\section{Introduction}

Codes over the ring of order six $Z_{6}$ have received some attention in the past due to their connection to euclidean lattices [11,13]. The main tool in this context is the Chinese Remainder Theorem (CRT) over the integers [8]. A classification technique for self-dual codes over this ring up to the permutation part of their monomial automorphism group was derived in [16], using the notion of double cosets in permutation groups. In a series of recent papers, the authors have studied self-orthogonal codes over nonunital rings of order $4[1,2,4,3]$. This is a major innovation in the domain of codes over rings, where only unital rings were used as alphabets till then $[18,19]$.

In the present paper, we combine these two strands of thought in the following way. We study self-orthogonal codes over two non-unital rings of order 6 , denoted here by $H_{32}$ and $H_{23}$. Both rings are semilocal with two maximal ideals of size two and three. There is a non multiplicative analogue of the CRT that allows to attach to any code over such a ring the ordered pair of a binary code and a ternary code. If the code is quasi self-dual (QSD) that is self-orthogonal of length $n$ and size $6^{n / 2}$, it can be shown that one of the two codes is self-dual and the other is a rate one-half code. Forgetting their multiplicative structure, we can regard the codes over either of these two non-unital rings as additive codes over $Z_{6}$, or, equivalently $Z_{6}$-linear codes. This simple observation allows us to use the Magma package for codes over rings [6] to compute weight distributions, and complete weight enumerators. We use the same classification methodology as that in [16] for codes over $Z_{6}$. We are able to classify QSD codes over these two rings up to length $n=8$.

The material is layed out in the following way. The next section collects the notations and notions needed for the rest of the paper. Section 3 is an exposition of the classification technique that we used. Section 4 contains some numerical data pertaining to that classification in modest length.

\section{Background material}

\subsection{Codes over fields}

Let $p$ be a prime. Denote by $w t(x)$ the Hamming weight of $x \in F_{p}^{n}$. The dual of a linear code $C$ over $F_{p}$ is denoted by $C^{\perp}$ and defined as

$$
C^{\perp}=\left\{y \in F_{p}^{n} \mid \forall x \in C,(x, y)=0\right\},
$$


where $(x, y)=\sum_{i=1}^{n} x_{i} y_{i}$, denotes the standard inner product. A code $C$ is self-orthogonal if it is included in its dual, i.e. $C \subseteq C^{\perp}$. A code is even if all its codewords have even weights. All binary self-orthogonal codes are even, but not all binary even codes are self-orthogonal. Two codes over $F_{p}$ are permutation equivalent if there is a permutation of coordinates that maps one to the other.

\subsection{Rings}

Let $C_{p}$ be the cyclic additive abelian group of order $p$ and $C_{p}(0)$ be the ring with additive group $C_{p}$ and trivial multiplication. We know (see for example [9,Lemma 2]) that, up to isomorphism, there are exactly two rings of order $p$, namely $Z_{p}$ and $C_{p}(0)$ and, if $p$ and $q$ are distinct primes, there are exactly four rings of order $p q$. These are $Z_{p q}, C_{p q}(0), H_{p q}:=Z_{p}+C_{q}(0)$, and $H_{q p}:=C_{p}(0)+Z_{q}$. The symbol + denotes the direct product of rings. Since the first two rings are well known, we consider in this paper the last two rings which are semi-local non-unital rings of order $p q$. In order to effectively construct codes, we restrict ourselves to the case $p=3$ and $q=2$. We denote these rings by

$$
H_{32}:=Z_{3}+C_{2}(0)=\left\langle a, b \mid 2 a=0,3 b=0, a^{2}=0, a b=0=b a, b^{2}=b\right\rangle,
$$

and

$$
H_{23}:=Z_{2}+C_{3}(0)=\left\langle a, b \mid 2 a=0,3 b=0, a^{2}=a, a b=0=b a, b^{2}=0\right\rangle .
$$

We denote by $c, d, e$ the remaining three elements, which we define as

$$
\begin{aligned}
c & =a+b \\
d & =2 b \\
e & =a+2 b .
\end{aligned}
$$

The addition tables of $H_{32}$ and $H_{23}$ are identical up to isomorphism and given by the following table 


\begin{tabular}{|c|c|c|c|c|c|c|}
\hline+ & 0 & $a$ & $b$ & $c$ & $d$ & $e$ \\
\hline 0 & 0 & $a$ & $b$ & $c$ & $d$ & $e$ \\
\hline$a$ & $a$ & 0 & $c$ & $b$ & $e$ & $d$ \\
\hline$b$ & $b$ & $c$ & $d$ & $e$ & 0 & $a$ \\
\hline$c$ & $c$ & $b$ & $e$ & $d$ & $a$ & 0 \\
\hline$d$ & $d$ & $e$ & 0 & $a$ & $b$ & $c$ \\
\hline$e$ & $e$ & $d$ & $a$ & 0 & $c$ & $b$ \\
\hline
\end{tabular}

The multiplication tables for respectively $H_{32}$ and $H_{23}$ are as follows.

\begin{tabular}{|l|l|l|l|l|l|l|}
\hline$\times$ & 0 & $a$ & $b$ & $c$ & $d$ & $e$ \\
\hline 0 & 0 & 0 & 0 & 0 & 0 & 0 \\
\hline$a$ & 0 & 0 & 0 & 0 & 0 & 0 \\
\hline$b$ & 0 & 0 & $b$ & $b$ & $d$ & $d$ \\
\hline$c$ & 0 & 0 & $b$ & $b$ & $d$ & $d$ \\
\hline$d$ & 0 & 0 & $d$ & $d$ & $b$ & $b$ \\
\hline$e$ & 0 & 0 & $d$ & $d$ & $b$ & $b$ \\
\hline
\end{tabular}

and

\begin{tabular}{|l|l|l|l|l|l|l|}
\hline$\times$ & 0 & $a$ & $b$ & $c$ & $d$ & $e$ \\
\hline 0 & 0 & 0 & 0 & 0 & 0 & 0 \\
\hline$a$ & 0 & $a$ & 0 & $a$ & 0 & $a$ \\
\hline$b$ & 0 & 0 & 0 & 0 & 0 & 0 \\
\hline$c$ & 0 & $a$ & 0 & $a$ & 0 & $a$ \\
\hline$d$ & 0 & 0 & 0 & 0 & 0 & 0 \\
\hline$e$ & 0 & $a$ & 0 & $a$ & 0 & $a$ \\
\hline
\end{tabular}

From these tables, we infer that these two rings are commutative, and without an identity element for the multiplication. They are semi-local with the two maximal ideals $J_{a}=\{0, a\}$, and $J_{b}=\{0, b, d\}$. Let $z \in\{23,32\}$. The following decomposition

$$
H_{z}=J_{a} \oplus J_{b},
$$

can be checked directly from the defining relations of $c, d, e$.

This alphabet decomposition induces a code decomposition as follows.

The code $C$ over $H_{z}$ can be written as a direct sum (in the sense of modules)

$$
C=a C_{a} \oplus b C_{b},
$$

where $C_{a}$ is a binary code and $C_{b}$ is a ternary code.

Denote by $\alpha_{a}: H_{z} \rightarrow F_{2}$ the map of reduction modulo $J_{a}$, and denote by $\alpha_{b}: H_{z} \rightarrow F_{3}$ the map of reduction modulo $J_{b}$, where $J_{a}$, and $J_{b}$ are viewed as additive groups. Thus $\alpha_{a}(a)=\alpha_{b}(b)=0$, and, by convention we choose $\alpha_{a}(b)=\alpha_{b}(a)=1$. These maps are extended in the natural way into maps from $H_{z}^{n}$ to $F_{2}^{n}$ (resp. from $H_{z}^{n}$ to $F_{3}^{n}$ ).

Thus, with these notations, we see that $C_{a}=\alpha_{b}(C)$ and $C_{b}=\alpha_{a}(C)$. 
Remark 1. These maps are additive morphisms but not ring morphisms. Still $C_{a}$ (resp. $C_{b}$ ) is a vector space over $F_{2}$ (resp. $F_{3}$ ) because $F_{2}$ (resp. $\left.F_{3}\right)$ is a prime field and additive subgroups of $F_{2}^{n}$ (resp. $F_{3}^{n}$ ) coincide with vector spaces over $F_{2}$ (resp. $F_{3}$ ).

\subsection{Weight Enumerators}

A linear $H_{z}$-code $C$ of length $n$ is an $H_{z}$-submodule of $H_{z}^{n}$. It can be described as the $H_{z^{-}}$span of the rows of a generator matrix. Two $H_{z^{-}}$ codes are permutation equivalent if there is a permutation of coordinates that maps one to the other.

An additive code $C$ of length $n$ over $Z_{6}$ is an additive subgroup of $Z_{6}^{n}$. It is an $Z_{6}$ sub-module of $Z_{6}^{n}$. By the CRT over the integers we can attach two codes to $C$ : a binary code $C_{2}$, and a ternary code $C_{3}$. We will write $C=C R T\left(C_{2}, C_{3}\right)$. Conversely, from every pair of a binary code $B$ and a ternary code $T$ of the same length a $Z_{6}$-code $C$ can be constructed by the formula $C=C R T(B, T)$.

If $C=a C_{a}+b C_{b}$ is an $H_{z}$-code, we can identify it with an additive $Z_{6}$-code given by $C=C R T\left(C_{a}, C_{b}\right)$. We use the Magma notation

$$
\left[<0,1>, \cdots,<i, A_{i}>, \cdots,<n, A_{n}>\right]
$$

for the weight distribution of a senary code, where $A_{i}$ is the number of codewords of Hamming weight $i$. The first index $i>0$ for which $A_{i}$ is nonzero is called the Hamming distance of the code. It is denoted by $d_{H}$. We will also require the complete weight enumerator in six variables cwe $_{C}$ of a senary code $C$ defined by

$$
\operatorname{cwe}_{C}\left(x_{0}, x_{1}, x_{2}, x_{3}, x_{4}, x_{5}\right)=\sum_{c \in C} \prod_{i=0}^{5} x_{i}^{n_{i}(c)},
$$

where $n_{i}(c)$ denotes for $i=0, \ldots, 5$, the number of coordinates $j$ for which $c_{j}=i$. Thus, if $n$ is the length of $C$, we have for all $c \in C$ the relation

$$
\sum_{i=0}^{5} n_{i}(c)=n
$$

We follow the notation of [15].

In view of the connection with lattices it makes sense to introduce Euclidean weight enumerator in one variable $e w e_{C}$ of a senary code $C$ 
defined as

$$
\operatorname{ewe}_{C}(y)=\sum_{c \in C} y^{w_{E}(c)},
$$

where the Euclidean weight of $x \in Z_{6}$ is defined as $w_{E}(x)=0,1,4,9$ if $x=0, \pm 1, \pm 2, \pm 3$ respectively. This notation is extended to vectors in the obvious way. The following connection with the complete weight enumerator is immediate from the definitions.

$$
\operatorname{ewe}_{C}(y)=\operatorname{cwe}\left(1, y, y^{4}, y^{9}, y^{4}, y\right) .
$$

The first exponent $i>0$ for which the coefficient of $y^{i}$ is nonzero is called the Euclidean distance of the code and is denoted by $d_{E}$.

For the next theorem we require some familiarity with lattices in the sense of the geometry of numbers $[7,11]$. The following result is the socalled Construction A of lattices from codes over $Z_{m}$ when $m=6$. For a proof see [7] for $m=2$, and [14] for $m=4$.

Theorem 1. If $C$ is a $Z_{6}$ code of length $n$ and size $6^{n / 2}$, then the lattice $A(C)$ given by

$$
\sqrt{6} A(C)=\bigcup_{c \in C}\left(c+6 Z^{n}\right)
$$

has determinant 1 and norm $\min \left(6, d_{E} / 6\right)$. If, furthermore, the code $C$ is self-dual, then $A(C)$ is unimodular.

Remark 2. It is proved in $[11,13]$ that $C=C R T(B, T)$ is self-dual iff both $B$ and $T$ are self-dual. Thus the $Z_{6}$-codes produced in this paper, in view of Lemma 1, and Lemma 2 are not always self-dual.

\subsection{Duality}

Define an inner product on $H_{z}^{n}$ as $(x, y)=\sum_{i=1}^{n} x_{i} y_{i}$.

The dual $C^{\perp}$ of $C$ is the module defined by

$$
C^{\perp}=\left\{y \in H_{z}^{n} \mid \forall x \in C,(x, y)=0\right\} .
$$

Thus the dual of a module is a module. A code is self-dual if it is equal to its dual.

A code $C$ is said to be self-orthogonal if

$$
\forall x, y \in C,(x, y)=0 .
$$

Clearly, $C$ is self-orthogonal iff $C \subseteq C^{\perp}$. 
Remark 3. Let the alphabet be $H_{32}$. The repetition code of length 2 is defined by $R_{2}:=\{00, a a, b b, c c, d d, e e\}$. We see that $R_{2}$ is self-orthogonal. Its dual contains also $b c, c b, e d$, de. (Note that the rows of $c$ and $b$ (resp. $d$ and e) are the same).Thus $R_{2}$ is not self-dual.

A code of length $n$ is said to be quasi self-dual (QSD) if it is selforthogonal and of size $2^{n}$.

Example: The code $R_{2}$ over either $H_{32}$ or $H_{23}$ is self-orthogonal of cardinality 6, hence it is QSD.

\section{Classification}

The following characterization result is easy but essential to understand the classification technique.

Lemma 1. Every $Q S D$ code $C$ of length $n$ over $H_{23}$ is of the form $a C_{a} \oplus b C_{b}$ where

1. $C_{a}$ is a self-dual binary code,

2. $C_{b}$ is an $[n, n / 2]$ ternary code.

In particular $n$ must be even.

Proof. The code $C$ is self-orthogonal iff $C_{a}$ is a self-orthogonal binary code because of the following identity

$$
\left(a x+b y, a x^{\prime}+b y^{\prime}\right)=a\left(x, x^{\prime}\right),
$$

where $x, x^{\prime}$ (resp. $y, y^{\prime}$ ) are arbitrary binary (resp. ternary) vectors of length $n$. Since by the QSD hypothesis $C=\left|C_{a}\right|\left|C_{b}\right|=6^{n / 2}$, we see that both $C_{a}$ and $C_{b}$ have dimension $n / 2$.

Lemma 2. Every QSD code $C$ of length $n$ over $H_{32}$ is of the form $a C_{a} \oplus b C_{b}$ where

1. $C_{b}$ is a self-dual ternary code,

2. $C_{a}$ is an $[n, n / 2]$ binary code.

In particular $n$ must be doubly even.

Proof. The proof of the first statement is analogous to the proof of Lemma 1, and is not written. To prove the second statement we use the 
fact that ternary self-dual codes only exist in doubly even lengths [15].

To classify QSD codes, we thus have to find all codes that are permutation equivalent to $a C_{a}+b C_{b}$ for a given pair $\left(C_{a}, C_{b}\right)$. This is a similar situation to the classification of self-dual codes over $Z_{p q}$ in [16], and we follow the method there. Here SDR stands for System of Distinct Representatives.

Theorem 2. Let $\left(C_{a}, C_{b}\right)$ be a pair of codes as defined in Lemma 1 or Lemma 2, with respective permutation groups $A$ and $B$. Then, the set

$$
S_{C_{a}, C_{b}}:=\left\{a C_{a}+b \sigma\left(C_{b}\right) \mid \sigma \text { runs over an SDR of } A \backslash S_{n} / B\right\}
$$

forms a set of inequivalent codes. In particular $\left|S_{C_{a}, C_{b}}\right|=\left|A \backslash S_{n} / B\right|$.

The next corollaries are immediate.

Corollary 1. Let $L_{a}$ be the set of all inequivalent self-dual binary codes of length $n$. Let $L_{b}$ be the set of all inequivalent $[n, n / 2]$ ternary codes. Then, the set of all QSD codes of length $n$ over $H_{23}$ is, up to coordinate permutation, the disjoint union $\underset{\substack{C_{a} \in L_{a} \\ C_{b} \in L_{b}}}{\bigsqcup} S_{C_{a}, C_{b}}$.

Corollary 2. Let $L_{b}$ be the set of all inequivalent self-dual ternary codes of length $n$. Let $L_{a}$ be the set of all inequivalent $[n, n / 2]$ binary codes. Then, the set of all QSD codes of length $n$ over $H_{32}$ is, up to coordinate permutation, the disjoint union $\coprod_{\substack{C_{a} \in L_{a} \\ C_{b} \in L_{b}}} S_{C_{a}, C_{b}}$.

To apply Corollary 1 effectively to classify QSD $H_{23}$-codes, we thus need to know two lists of codes, for a given length $n$.

1. An SDR of equivalence classes of self-dual $[n, n / 2]$ binary codes,

2. an SDR of equivalence classes of ternary $[n, n / 2]$ codes.

To apply Corollary 2 effectively to classify QSD $H_{32}$-codes, we thus need to know two lists of codes, for a given length $n$.

1. An SDR of equivalence classes of self-dual $[n, n / 2]$ ternary codes,

2. an SDR of equivalence classes of binary $[n, n / 2]$ codes. 
The first type of list can be found in [12]. The second type of list can be established by using the method in $[5,10]$.

The classification algorithm for QSD $H_{23}$-codes can be described as follows. Given an even length $n \geq 1$, do the following steps.

1. Write a list $L_{a}$ of self-dual $[n, n / 2]$ binary codes.

2. Write a list $L_{b}$ of ternary $[n, n / 2]$ codes.

3. For all pairs $\left(C_{a}, C_{b}\right)$ in $L_{a} \times L_{b}$ do the following.

1. Compute the permutation groups $A$ and $B$ of $C_{a}$ and $C_{b}$ respectively.

2. Find a list $\sigma_{1}, \ldots, \sigma_{s}$ of representatives of $A \backslash S_{n} / B$.

3. For $i=1$ to $s$ output $a C_{a}+b \sigma_{i}\left(C_{b}\right)$.

A similar description holds for QSD $H_{32}$-codes.

1. Write a list $L_{b}$ of self-dual $[n, n / 2]$ ternary codes.

2. Write a list $L_{a}$ of binary $[n, n / 2]$ codes.

3. For all pairs $\left(C_{a}, C_{b}\right)$ in $L_{a} \times L_{b}$ do the following.

1. Compute the permutation groups $A$ and $B$ of $C_{a}$ and $C_{b}$ respectively.

2. Find a list $\sigma_{1}, \ldots, \sigma_{s}$ of representatives of $A \backslash S_{n} / B$.

3. For $i=1$ to $s$ output $a C_{a}+b \sigma_{i}\left(C_{b}\right)$.

\section{Numerical results}

All the computations needed for this section were performed in Magma [6], except for the length 8 where we used Sage [17]. The Euclidean distance of the codes below is computed by inspection of the Euclidean weight enumerator, which is, most of the time, too long to be displayed. In the following sections $Z_{6}$-codes are classified up to coordinate permutation, which is a weaker form of equivalence than that used in $[12,13,16]$ where the permutation part of the monomial automorphism group of the codes is authorized. Thus, we find seventeen euclidean self-dual $Z_{6}$-codes for $n=8$, where only five are found in $[12,16]$. In general all the self-dual codes mentioned below are self-dual for the standard inner product over $Z_{6}$ like in $[12,13,16]$. 


\subsection{The Ring $H_{32}$}

The bijective correspondence $H_{32} \leftrightarrow Z_{6}$ is given informally by

$$
0=0, e=1, b=2, a=3, d=4, c=5 .
$$

\subsubsection{Length 4 (13 codes)}

The main properties are summarized in the following table.

\begin{tabular}{|c|c|c|c|c|}
\hline \# codes & 4 & 6 & 1 & 2 \\
\hline$d_{H}$ & 1 & 1 & 2 & 2 \\
\hline$d_{E}$ & 6 & 3 & 6 & 3 \\
\hline
\end{tabular}

There is a unique self-dual $Z_{6}$-code of length 4 . Its ewe is

$$
y^{36}+10 y^{18}+16 y^{12}+8 y^{6}+1 .
$$
$Z^{4}$.

This is consistent with [16, Table 2]. Construction A yields the lattice

\subsubsection{Length 8 (11 615 codes)}

The Hamming distance distribution is summarized in the following table.

\begin{tabular}{|c|c|c|c|}
\hline \# codes & 4516 & 6365 & 743 \\
\hline$d_{H}$ & 1 & 2 & 3 \\
\hline
\end{tabular}

There are seventeen self-dual codes, eleven of Hamming weight 2 and Euclidean weight 6 , and six of Hamming weight 3 and Euclidean weight 12. The six codes of Euclidean distance 12 yield the lattice $E_{8}$ by Theorem 1, the unique Type II lattice in dimension 8 [7].

yields the lattice $Z^{8}$ by construction $\mathrm{A}$.

\subsection{The Ring $H_{23}$}

The bijective correspondence $H_{23} \leftrightarrow Z_{6}$ is given informally by

$$
0=0, e=1, b=2, a=3, d=4, c=5 .
$$

\subsubsection{Length 2 (two codes)}

We obtain two codes one with generator matrix (13), $d_{H}=1, d_{E}=4$, and the other one with generator matrix $(11), d_{H}=2, d_{E}=2$. 


\subsubsection{Length 4 (fourteen codes)}

The metric properties are summarized in the following table.

\begin{tabular}{|c|c|c|c|c|c|}
\hline \# codes & 2 & 3 & 3 & 5 & 1 \\
\hline$d_{H}$ & 1 & 1 & 2 & 2 & 2 \\
\hline$d_{E}$ & 2 & 4 & 2 & 4 & 6 \\
\hline
\end{tabular}

The unique code with $d_{E}=6$ is the self-dual code obtained in $\S 4.1 .1$.

\subsubsection{Length 6 (162 codes)}

The metric properties are summarized in the following table.

\begin{tabular}{|c|c|c|c|c|c|}
\hline \# codes & 16 & 34 & 25 & 56 & 31 \\
\hline$d_{H}$ & 1 & 1 & 2 & 2 & 2 \\
\hline$d_{E}$ & 2 & 4 & 2 & 4 & 6 \\
\hline
\end{tabular}

\subsubsection{Length 8 (10447 codes)}

The metric properties are summarized in the following table.

\begin{tabular}{|c|c|c|c|c|c|c|c|c|c|c|c|c|}
\hline \# codes & 209 & 1797 & 509 & 3690 & 11 & 3179 & 416 & 481 & 117 & 6 & 27 & 5 \\
\hline$S D$ & $N$ & $N$ & $N$ & $N$ & $Y$ & $N$ & $N$ & $N$ & $N$ & $Y$ & $N$ & $N$ \\
\hline$d_{H}$ & 1 & 1 & 2 & 2 & 2 & 2 & 2 & 3 & 3 & 3 & 4 & 4 \\
\hline$d_{E}$ & 2 & 4 & 2 & 4 & 6 & 6 & 8 & 4 & 8 & 12 & 4 & 8 \\
\hline
\end{tabular}

Like for codes over $H_{32}$, there are 17 self-dual codes, 11 of Hamming weight 2 and Euclidean weight 6 , and 6 of Hamming weight 3 and Euclidean weight 12 . The 6 codes of Euclidean distance 12 yield the lattice $E_{8}$ by Theorem 1, the unique Type II lattice in dimension 8 [7].

\section{Acknowledgement:}

The authors are indebted to Prof. David Kohel for helping them with computations in the computer package Sage [17].

\section{References}

[1] A. Alahmadi, A. Alkathiry, A. Altassan, A. Bonnecaze, H. Shoaib, and P. Solé, "The build-up construction of quasi self-dual codes over a nonunital ring", Journal of algebra and applications, accepted preprint, 2020. [On line]. Available: https://bit.ly/38HMJ8t 
[2] A. Alahmadi, A. Alkathiry, A. Altassan, A. Bonnecaze, H. Shoaib, and P. Solé, "The build-up construction of quasi self-dual codes over a commutative non-unital ring", submitted preprint, 2020.

[3] A. Alahmadi, A. Altassan, W. Basaffar, A. Bonnecaze, H. Shoaib, and P. Solé, "Quasi Type IV codes over a non-unital ring", submitted preprint, 2020. [On line]. Available: https://bit.ly/3gozCvo

[4] A. Alahmadi, A. Altassan, W. Basaffar, A. Bonnecaze, H. Shoaib, and P. Solé, "Type IV codes over a non-unital ring", Journal of algebra and applications, accepted preprint, 2020. [On line]. Available: https://bit.ly/3f5D8ue

[5] A. Betten, M. Braun, H. Fripertinger, A. Kerber, A. Kohnert and A. Wassermann, Error correcting-linear codes. Berlin: Springer, 2006, doi: 10.1007/3-540-31703-1

[6] Computational Algebra Group. and University of Sydney, "Magma computational algebra system", Magma computer algebra, 2010. [Online]. Available: http://magma.maths.usyd.edu.au/magma/

[7] J. H. Conway and N. J. A. Sloane, Sphere packings, lattices and groups, 2nd ed. New York, NY: Springer, 2003, doi: 10.1007/978-1-47572249-9

[8] S. T. Dougherty, M. Harada, and P. Solé, "Self-dual codes over rings and the chinese remainder theorem", Hokkaido mathematical journal, vol. 28, no. 2, pp. 253-283, 1999, doi: 10.14492/hokmj/1351001213

[9] B. Fine, "Classification of Finite rings of order p2"; Mathematics magazine, vol. 66, no. 4, pp. 248-252, Oct. 1993, doi: 10.1080/0025570X.1993.11996133

[10] H. Fripertinger, "Enumeration of isometry classes of linear (n, k)codes over GF (q), SYMMETRICA", Bayreuther mathematische schriften, vol. 49, pp. 215-223, 1999. [On line]. Available: https://bit.ly/31QmYRC

[11] M. Harada and M. Kitazume, " $\mathrm{Z}_{6}$-code constructions of the Leech latttice and the Niemeier lattices", European journal of combinatory, vol. 23, no. 5, pp. 573-581, Jul. 2002 , doi: 10.1006/eujc.2002.0557 
[12] M. Harada and A. Munemasa, "Database of self-dual codes", Tohoku University Graduate School of Information Science, Department of Mathematics/Center for Pure and Applied Mathematics, 15-Nov-2007. [Online]. Available: http://www.math.is.tohoku.ac.jp/ munemasa/ selfdualcodes.htm.

[13] M. Harada and A. Munemasa, "On the classification of self-dual open image in new window $\mathbb{Z}_{\mathrm{k}}$-codes", in Cryptography and coding, M. G. Parker, Ed. Berlin: Springer, 2009, pp. 78-90, doi: 10.1007/978-3642-10868-6_6

[14] W. C. Hufman and V. Pless, Fundamentals of error correcting codes, Cambridge: Cambridge University Press, 2003, doi: 10.1017/CBO9780511807077

[15] F. J. MacWilliams and N. J. A. Sloane, Eds., The theory of error-correcting codes, North-Amsterdam: North-Holland, 1977, doi: 10.1016/s09246509(08)x7030-8

[16] Y-H. Park, "The classification of self-dual modular codes", Finite fields and their applications, vol. 17, no. 5, pp. 442-460, Sep. 2011, doi: 10.1016/j.ffa.2011.02.010

[17] SageMath Mathematical Software System, "Sage", 2007. [Online]. Available: https://www.sagemath.org/

[18] M. Shi, A. Alahmadi, and P. Solé, Codes and rings: theory and practice, Cambridge, MA: Academic Press, 2017, doi: 10.1016/C2016-0-0442 9-7

[19] P. Solé, Ed., Codes over rings, Singapore: World Scientific, 2008, doi: $10.1142 / 7140$. 